\title{
Comparison LEACH and HEED Cluster Based protocol for Wireless Senser Network
}

\author{
${ }^{1}$ Pratik R. chavda, ${ }^{2}$ Prof Paresh kotak \\ ${ }^{I}$ Department Computer Science and Engineering University of GTU, Rajkot, 360005, India, \\ ${ }^{2}$ Principal A.V.PAREKH Technical Institute Rajkot.
}

\begin{abstract}
In the present paper wireless senser network for hierarchical network protocol of LEACH and HEED this is theory concept that how to data gathering. Our protocol aims to dynamically change the data Gathering period according to the Gathering quality. The timing scheme will find the minimum Gathering period that satisfies the sink's request. This protocol is work on this and how to easy maintain from both of them. The LEACH (low energy adaptive clustering hierarchy) protocol presented in is an elegant solution where clusters are formed to fuse data before transmitting to the base station. We propose a new energy-efficient approach for clustering nodes in adhoc sensor networks. Based on this approach, we present a protocol, HEED (Hybrid Energy-Efficient Distributed clustering), that periodically selects cluster heads according to a hybrid of their residual energy and a secondary parameter, such as node proximity to its neighbors or node degree. In both routing protocol we differ which is batter to use and give most of the point to cover in this paper.
\end{abstract}

Key words-Data Gathering, LEACH, HEED.

\section{Introduction}

Data Gathering is a process of aggregating the sensor data using Gathering approaches. The general data Gathering algorithm works as shown in the below figure. The algorithm uses the sensor data from the sensor node and then aggregates the data by using some Gathering algorithms such as centralized approach, LEACH(low energy adaptive clustering hierarchy),TAG(Tiny Gathering) etc. This Gathered data is transfer to the sink node by selecting the efficient path.

There are many types of Gathering techniques are present some of them are listed below.

Centralized Approach: This is an address centric approach where each node sends data to a central node via the shortest possible route using a multihop wireless protocol. The sensor nodes simply send the data packets to a leader, which is the powerful node. The leader gathered the data which can be queried. Each intermediate node has to send the data packets addressed to leader from the child nodes. So a large number of messages have to be transmitted for a query in the best case equal to the sum of external path lengths for each node.

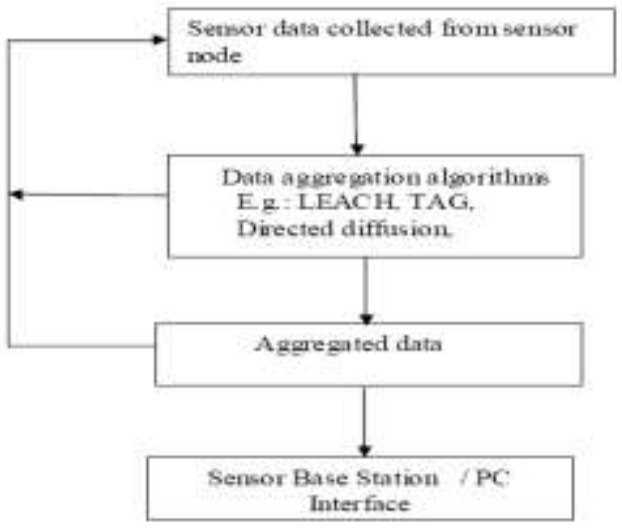

Fig 1.1: General architecture of the data Gathering algorithm

In-Network Gathering [7]: In-network Gathering is the global process of gathering and routing information through a multi-hop network, processing data at intermediate nodes with the objective of reducing resource consumption (in particular energy), thereby increasing network lifetime. There are two approaches for innetwork Gathering: with size reduction and without size reduction. In-network Gathering with size reduction refers to the process of combining \& compressing the data packets received by a node from its neighbors in order to reduce the packet length to be transmitted or forwarded towards sink. In-network Gathering without 
size reduction refers to the process merging data packets received from different neighbors in to a single data packet but without processing the value of data.

Tree-Based Approach [8]: In the tree-based approach perform Gathering by constructing a Gathering tree, which could be a minimum spanning tree, rooted at sink and source nodes are considered as leaves. Each node has a parent node to forward its data. Flow of data starts from leaves nodes up to the sink and Therein the Gathering done by parent nodes.

Cluster-Based Approach [6]: In cluster-based approach, whole network is divided in to several clusters. Each cluster has a cluster-head which is selected among cluster members. Cluster heads do the role of aggregator which aggregate data received from cluster members locally and then transmit the result to sink.

\section{Requirements of Private Data Gathering}

Protecting the data privacy in many wireless sensor network applications is a major concern. The following criteria summarize the desirable characteristics of a private data Gathering scheme:

1) Privacy: Each node's data should be only known to itself. Furthermore, the private data Gathering scheme should be able to handle to some extent attacks and collusion among compromised nodes. When a sensor network is under a malicious attack, it is possible that some nodes may collude to uncover the private data of other node(s). Furthermore, wireless links may be eavesdropped by attackers to reveal private data. A good private data Gathering scheme should be robust to such attacks.

2) Efficiency: The goal of data Gathering is to reduce the number of messages transmitted within the sensor network, thus reduce resource and power usage. Data Gathering achieves bandwidth efficiency by using innetwork processing. In private data Gathering schemes, additional overhead is introduced to protect privacy. However, a good private data Gathering scheme should keep that overhead as small as possible.

3) Accuracy: An accurate Gathering of sensor data is desired, with the constraint that no other sensors should know the exact value of any individual sensor. Accuracy should be a criterion to estimate the performance of private data Gathering schemes.

\section{Hierarchical networks}

A flat network can result in excessive communication and computation burden at the sink node resulting in a faster depletion of its battery power. The death of the sink node breaks down the functionality of the network. Hence, [9] in view of scalability and energy efficiency, several hierarchical data aggregation approaches have been proposed. Hierarchical data aggregation involves data fusion at special nodes, which reduces the number of messages transmitted to the sink. This improves the energy efficiency of the Network. In the rest of this subsection, we describe the different hierarchical data aggregation protocols and highlight their main Advantages and limitations.

\section{Data aggregation in cluster based networks}

In energy constrained sensor networks of large size, it is inefficient for sensors to transmit the data directly to the sink. In such scenarios, sensors can transmit data to a local aggregator[14]

or cluster head which aggregates data from all the sensors in its cluster and transmits the concise digest to the sink. This results in significant energy savings for the energy constrained sensors. Figure shows a cluster based sensor network organization. The cluster heads can communicate with the sink directly via long range transmissions or multi hopping through other cluster heads. Recently, several cluster based network organization and data aggregation protocols have been proposed. In this section we discuss three such protocols viz., Low Energy Adaptive Clustering Hierarchy (LEACH), Hybrid Energy Efficient Distributed Clustering Approach (HEED) and clustered diffusion with dynamic data aggregation (CLUDDA).

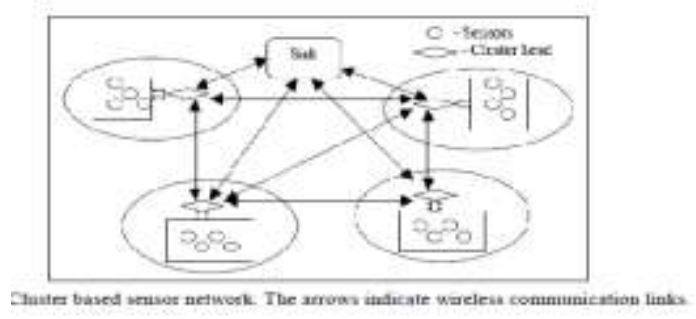




\section{Related Work}

Data aggregation in cluster based networks

Low Energy Adaptive Clustering Hierarchy protocol (LEACH) LEACH [2],[4],[5] is a kind of cluster-based routing protocols, which includes distributed cluster formation. LEACH randomly selects a few sensor nodes as cluster heads (CHs) and rotates this role to evenly distribute the energy load among the sensors in the network. The idea is to form clusters of the sensor nodes based on the received signal strength and use local cluster heads as routers to the sink. In $\mathrm{LEACH}$, the $\mathrm{CH}$ nodes compress data arriving from nodes that belong to the respective cluster, and send an aggregated packet to the BS in order to reduce the amount of information that must be transmitted to the BS. LEACH uses a TDMA/code-division multiple access (CDMA) MAC to reduce intercluster and intra-cluster collisions. All the data processing such as data fusion and aggregation are local to the cluster. The operation of LEACH is done into two phases, the setup phase and the steady state phase. In setup phase the clusters are organized and $\mathrm{CHs}$ are selected. Cluster heads change randomly over time in order to balance the energy dissipation of nodes. This decision is made by the node choosing a random number between 0 and 1 . The node becomes a cluster head for the current round if the number is less than the following threshold value $T l(n)$,

$$
T_{1}(n)=\frac{P}{1-P *\left(r \bmod \frac{1}{P}\right)},
$$

LEACH clustering is shown in formula. In the steady state phase, the actual data transfer to the BS takes place. The duration of the steady state phase is longer than the duration of the setup phase in order to minimize overhead. During the steady state phase, the sensor nodes can begin sensing and transmitting data to the CHs. The $\mathrm{CH}$ node, after receiving all the data, aggregates it before sending it to the BS. After a certain time, which is determined a priori, the network goes back into the setup phase again and enters another round of selecting new CHs. Each cluster communicates using different CDMA codes to reduce interference from nodes belonging to other clusters.

The major characteristics of this Protocol are as follow:

It rotates the cluster heads in a randomized fashion to achieve balanced energy consumption, Sensors have synchronized clocks so that they know the beginning of a new cycle, Sensors do not need to know location or distance information.

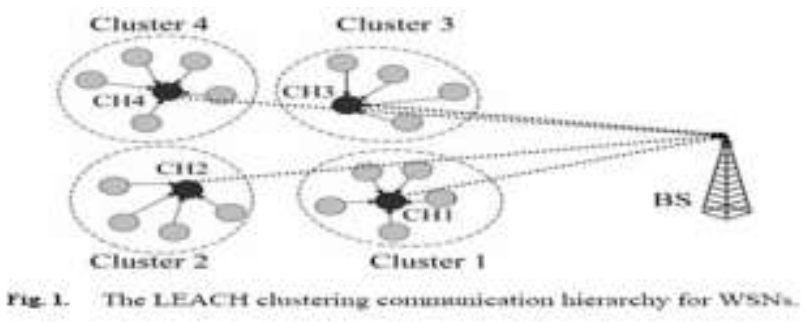

There are some drawbacks of this protocol such as:

LEACH uses single-hop routing where each node can transmit directly to the cluster-head and the sink. Therefore, it is not applicable to networks deployed in large regions. The idea of dynamic clustering brings extra overhead, e.g. head changes, advertisements etc., which may decrease the gain in energy consumption. Random election of $\mathrm{CH},[10]$ hence there is Possibility that all $\mathrm{CHs}$ will be concentrated in same area. The protocol assumes that all nodes begin with the same amount of energy capacity in each election round, assuming that being a $\mathrm{CH}$ consumes approximately the same amount of energy for each node.

This protocol is most suited for constant monitoring such as monitor machinery for fault detection and diagnosis.

Hybrid, Energy-Efficient Distributed Clustering (HEED) HEED [11] excellent cluster-based protocol it elect CHs based on residual energy and node degree or density of nodes as a metric for cluster selection to achieve power balancing, which is a rational improvement compared with LEACH. In HEED, the proposed algorithm periodically selects $\mathrm{CHs}$ according to a combination of two clustering parameters. The primary parameter is their residual energy of each sensor node and the secondary parameter is the intra-cluster communication cost as a function of cluster density. The primary parameter is used to probabilistically select an initial set of $\mathrm{CHs}$ while the secondary parameter is used for breaking ties. HEED was proposed with four primary goals namely,

(i) Prolonging network lifetime by distributing energy consumption,

(ii) Terminating the clustering process within a constant number of iterations,

(iii) Minimizing control overhead,

(iv) Producing well-distributed CHs and compact clusters. 


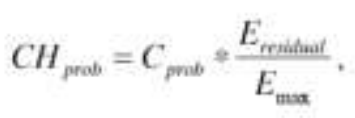

In HEED, the clustering process at each sensor node requires several rounds. Every round is long enough to receive messages from any neighbor within the cluster range. The parameter Cprob is only used to limit the initial $\mathrm{CH}$ announcements and has no direct impact on the final cluster structure. In HEED, each sensor node sets the probability CHprob of becoming a $\mathrm{CH}$ as follows. Where Eresidual is the estimated current residual energy in this sensor node and Emax is the maximum energy corresponding to a fully charged battery, which is typically identical for homogeneous sensor nodes. The $C H p r o b$ value must be greater than a minimum threshold pmin. A CH is either a tentative $\mathrm{CH}$, if its $\mathrm{CHprob}$ is $<1$, or a final $\mathrm{CH}$, if its $\mathrm{CHprob}$ has reached 1.During each round of HEED, every sensor node that never heard from a $\mathrm{CH}$ elects itself to become a $\mathrm{CH}$ with probability CHprob. The newly selected $\mathrm{CHs}$ are added to the current set of $\mathrm{CHs}$. If a sensor node is selected to become a $\mathrm{CH}$, it broadcasts an announcement message as a tentative $\mathrm{CH}$ or a final $\mathrm{CH}$. A sensor node hearing the $\mathrm{CH}$ list selects the $\mathrm{CH}$ with the lowest cost from this set of CHs. Every node then doubles its $\mathrm{CHprob}$ and goes to the next step. If a node completes the HEED execution without electing itself to become a $\mathrm{CH}$ or joining a cluster, it announces itself as a final $\mathrm{CH}$.Atentative $\mathrm{CH}$ node can become a regular node at a later iteration if it hears from a lower cost $\mathrm{CH}$. Here, a node can be selected as a $\mathrm{CH}$ at consecutive clustering intervals if it has higher residual energy with lower cost. The important features of this protocol are as follows: HEED distribution of energy extends the lifetime of the nodes within the network thus stabilizing the neighboring node. HEED does not require special node capabilities, such as location-awareness HEED does not make assumptions about node distribution the nodes also automatically update their neighbor sets in multi-hop networks by periodically sending and receiving messages. It operates correctly even when nodes are not synchronized. The nodes only require local (neighborhood) information to form the clusters.

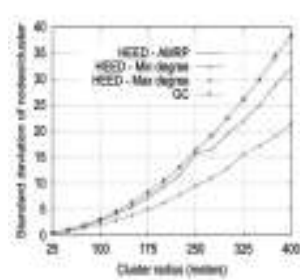

(b)

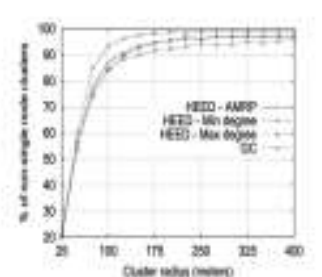

A

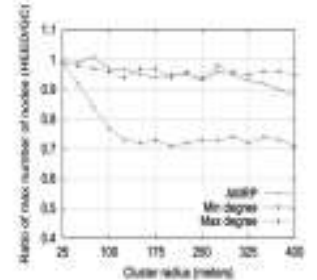

$|c|$

Characteristics of clusters. (a) Standard deviation of the number of nodes/cluster. (b) Percentage of non-singlenode clusters. (c) Ratio of Maximum number of nodes in a HEED cluster to a GC cluster.

There are some disadvantages of this protocol such as:

The random selection of the cluster heads may cause higher communication overhead for: The ordinary member nodes in communicating with their corresponding cluster head, the cluster heads in establishing the communication among them, or between a cluster head and a base station. The periodic cluster head rotation or election needs extra energy to rebuild clusters. This protocol is most suitable for prolonging the network lifetime rather than for the entire needs of WSN.

A. Compare Both The Protocol And Its Charactristic

\section{Work Analysis}

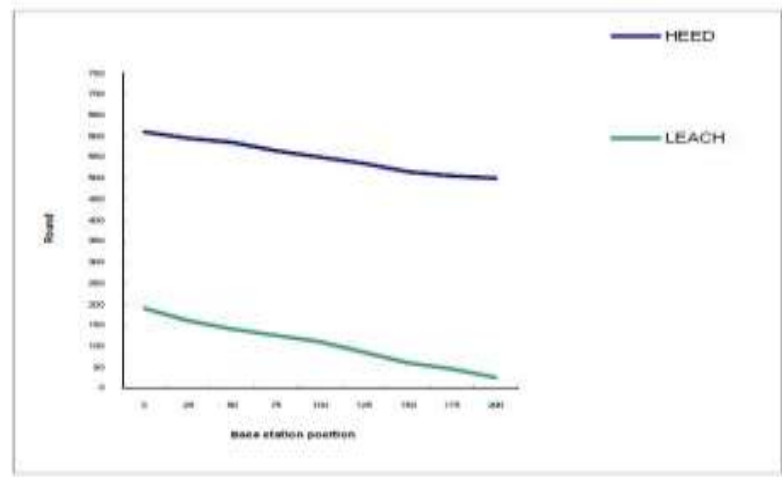

Figure 4. sink node position and network lifetime. 
Figure shows that the lifetime of the network between LEACH and HEED, protocols vary with the number of nodes from 100 to 500. Well, this may due to the following reasons. First, alternating the role of $\mathrm{CH}$ can balance energy consumption among these clusters member. Second, our fuzzy spanning tree is effective in prolonging the lifetime of $\mathrm{CHs}$. Third, our $\mathrm{CH}$ election algorithm more energy efficient, as a result extend the network lifetime.

We compare our HEED clustering to a generalized LEACH approach in which two features are added to the application-specific LEACH protocol, described in [8]. First, the routing protocol is assumed to propagate node residual energy throughout the network. Although this approach requires extensive message exchange (for residual energy information), it selects better cluster heads than the original LEACH and, thus, prolongs the network lifetime (this approach was proposed in the code released by the authors of [8]).

Comparison Results of LEACH and HEED Protocols:

\begin{tabular}{|l|l|l|l|l|l|l|}
\hline $\begin{array}{l}\text { Protocol } \\
\text { Name }\end{array}$ & $\begin{array}{l}\text { Energy } \\
\text { Efficienc } \\
\mathbf{y}\end{array}$ & $\begin{array}{l}\text { Cluster } \\
\text { Stabilit } \\
\mathbf{y}\end{array}$ & Scalability & $\begin{array}{l}\text { Delivery } \\
\text { Delay }\end{array}$ & $\begin{array}{l}\text { Load } \\
\text { Balancing }\end{array}$ & $\begin{array}{l}\text { Algorithm } \\
\text { Complexit } \\
\mathbf{y}\end{array}$ \\
\hline LEACH & very low & $\begin{array}{l}\text { modera } \\
\text { te }\end{array}$ & very low & very small & moderate & low \\
\hline HEED & moderate & high & moderate & moderate & moderate & moderate \\
\hline
\end{tabular}

The advantages of the HEED protocol are as follows: (1) It is a fully distributed clustering method that benefits from the use of the two important parameters for $\mathrm{CH}$ election; (2) Low power levels of clusters promote an increase in spatial reuse while high power levels of clusters are required inter-cluster communication. This provides uniform $\mathrm{CH}$ distribution across the network and load balancing; (3) Communications in a multi-hop fashion between $\mathrm{CHs}$ and the BS promote more energy conservation and scalability in contrast with the single-hop fashion, i.e., long-range communications directly from $\mathrm{CHs}$ to the sink, in the LEACH protocol [16]. However, there are some limitations with HEED as follows: (1) the uses of tentative $\mathrm{CHs}$ that do not become final $\mathrm{CHs}$ leave some uncovered nodes. As per HEED implementation, these nodes are forced to become a $\mathrm{CH}$ and these forced $\mathrm{CHs}$ may be in range of other $\mathrm{CHs}$ or may not have any member associated with them. As a result, more $\mathrm{CHs}$ are generated than the expected number and this also accounts for unbalanced energy consumption in the network [17]; (2) Similar to LEACH, performing of clustering in each round imposes significant overhead in the network. This overhead causes noticeable energy dissipation which results in decreasing the network lifetime; (3) HEED suffers from a consequent overhead since it needs several iterations to form clusters. At each iteration, a lot of packets are broadcast. (4) Some CHs, especially near the sink, may die earlier because these $\mathrm{CHs}$ have more work load, and the hot spot will come into being in the network $[17,18]$.

\section{Conclusion And Future Scope:}

Finally it is concluded from the survey that, still it is needed to find more scalable, energy efficient and stable clustering scheme, for data gathering in wireless sensor networks. After the comparing we review that the HEED routing protocol is more energy efficient routing protocol for wireless sensor network as comparisons to LEACH protocol in the form of energy consumption and cost of sensor nodes. Further in future we use the movable nodes to compare and analyzed these protocols.

\section{References}

[1] J. Al-Karaki, and A. Kamal, .Routing Techniques in Wireless Sensor Networks: A Survey., IEEE Commun-ications Magazine, vol 11, no. 6, Dec. 2004,pp. 6-28.

[2] W. Heinzelman, A. Chandrakasan and H. Balakrishnan,"Energy-Efficient CommunicationProtocol for Wireless Mi- crosensor Networks," Proceedings of the 33rd Hawaii International Conference on System Sciences (HICSS '00), January 2000.

[3] W.R. Heinzelman, A. Chandrakasan, and H. Balakrishna

[4] K. Dasgupta, K. Kalpakis, and P. Namjoshi, "An Efficient Clustering-based Heuristic for Data Gathering and Gathering in Sensor Networks", IEEE 2003.

[5] E. Fasolo, M. Rossi, J. Widmer, and M. Zorzi, "In- Network Gathering Techniques for Wireless Sensor Networks: A Survey", IEEE Wireless communication 2007.

[6] W. Heinzelman, A. Chandrakasan, and H. Balakrishnan, "An Application-Specific Protocol Architecture for Wireless Microsensor Networks,” IEEE Trans. Wireless Comm., vol. 1, no. 4, pp. 660- 670, Oct. 2002.

[7] N.M. Elshakankiri, N. M. Moustafa and Y. H. Dakroury, -Energy Efficient Routing Protocol for Wireless Sensor Network $\|$ in IEEE InternationalConference on pp. 393-398,December 2008.

[8] J. Al-Karaki, and A. Kamal, .Routing Techniques in Wireless Sensor Networks: A Survey., IEEE Commun-ications Magazine, vol 11, no. 6, Dec. 2004,pp. 6-28. 11.)Jamil Ibriq and lmad Mahgoub, . Cluster-based Routing in Wireless Sensor Networks: Issues and Challe- nges., SPECTS.04, pp. 759-766.

[9] W. R. Heinzelman, "Application-specific protocol architectures for wireless networks", PhD Thesis, Massachusetts Institute of Technology, June 2000. 
[10] C. Intanagonwiwat, R. Govindan and D. Estrin, "Directed Diffusion: A Scalable and robust communication paradigm for sensor networks," Proceedings of the Sixth Annual International Conference on Mobile Computing and Networking (MobiCOM '00), August 2000.

[11] O. Younis and S. Fahmy, "HEED: a hybrid, energy-efficient, distributed clustering approach for ad hoc sensor networks," IEEE Transactions on Mobile Computing, vol .3, no. 4, Dec 2004, pp. 366-79.

[12] M. Ding, X. Cheng and G. Xue, "Aggregation tree construction in sensor networks," 2003 IEEE 58th Vehicular Technology Conference, vol.4, no.4, October 2003, pp 2168-2172.

[13] Taheri, H.; Neamatollahi, P.; Younis, O.M.; Naghibzadeh, S.; Yaghmaee, M.H. An energy-aware distributed clustering protocol in wireless sensor networks using fuzzy logic. Ad Hoc Netw. 2012, 10,1469 \pm 1481 .

[14] Aslam, N.; Phillips, W.; Robertson, W.; Sivakumar, S. A multi-criterion optimization technique for energy efficient cluster formation in wireless sensor networks. Inf. Fusion 2011, 12, 202 \pm 212 .

[15] Poonguzhali, P.K. Energy Efficient Realization of Clustering Patch Routing Protocol in Wireless Sensors Network. In Proceedings of the 2nd International Conference on Computer Communication and Informatics, Coimbatore, Tamilnadu, India, $10 \pm 12$ January 2012; pp. 10 \pm 12 . 